\title{
Lutein intake at the age of 1 year and cardiometabolic health at the age of 6 years: the Generation R Study
}

\author{
Elisabeth T. M. Leermakers ${ }^{1,2}$, Jessica C. Kiefte-de Jong ${ }^{2,3 *}$, Albert Hofman ${ }^{2}$, Vincent W. V. Jaddoe ${ }^{1,2,4}$ and \\ Oscar H. Franco ${ }^{1,2}$ \\ ${ }^{1}$ Generation R Study Group, Department of Epidemiology, Erasmus MC, University Medical Center, 300O CA, Rotterdam, \\ The Netherlands \\ ${ }^{2}$ Department of Epidemiology, Erasmus MC, University Medical Center, 3000 CA, Rotterdam, The Netherlands \\ ${ }^{3}$ Faculty Campus The Hague, Leiden University College, 2595 DG The Hague, The Netherlands \\ ${ }^{4}$ Department of Pediatrics, Erasmus MC, University Medical Center, 3000 CA, Rotterdam, The Netherlands
}

(Submitted 4 February 2015 - Final revision received 16 June 2015 - Accepted 25 June 2015)

\section{Abstract}

Lutein is a carotenoid with strong antioxidant properties. Previous studies in adults suggest a beneficial role of lutein on cardiometabolic health. However, it is unknown whether this relation also exists in children; therefore, we aimed to assess the relation between lutein intake at 13 months of age and cardiometabolic outcomes at the age of 6 years. We included 2044 Dutch children participating in a population-based prospective cohort study. Diet was measured at 13 months of age with an FFQ. Lutein intake was standardised for energy and $\beta$-carotene intake. Blood pressure, anthropometrics, serum lipids and insulin were measured at the age of 6 years. Dual-energy X-ray absorptiometry was performed to measure total and regional fat and lean mass. A continuous cardiometabolic risk factor score was created, including the components body fat percentage, blood pressure, insulin, HDL-cholesterol and TAG. Age- and sex-specific standard deviation scores were created for all outcomes. Multivariable linear regression was performed, including socio-demographic and lifestyle variables. Median (energy-standardised) lutein intake was $1317 \mathrm{mcg} / \mathrm{d}(95 \% \mathrm{range} 87,6069 \mathrm{mcg} / \mathrm{d}$ ). There were no consistent associations between lutein intake at 13 months and anthropometrics and body composition measures at 6 years of age. In addition, lutein intake was not associated with a continuous cardiometabolic risk factor score, nor was it associated with any of the individual components of the cardiometabolic risk factor score. Results from this large population-based prospective cohort study do not support the hypothesis that lutein intake early in life has a beneficial role for later cardiometabolic health.

\section{Key words: Carotenoids: Lutein: Body composition: Cardiometabolic health: Epidemiology: Children}

Lutein is a non-provitamin A carotenoid that is present in many commonly consumed fruits and vegetables ${ }^{(1)}$. Due to ingestion of these foods by animals, lutein is also present in animal products such as eggs and dairy products. As an antioxidant, lutein has the potential to protect against oxidative stress by quenching lipid peroxide radicals, and could therefore protect an individual from CVD and metabolic diseases ${ }^{(1)}$.

Previous literature has shown associations between higher lutein intake or blood levels and a decreased risk of metabolic syndrome $^{(2-6)}$. In particular, lutein has been shown to be inversely related to waist circumference ${ }^{(6,7)}$ and $\mathrm{BMI}^{(5,7)}$. Nevertheless, reverse causation could apply as lutein is fatsoluble, and thus adiposity might lead to lower levels of lutein due to lutein absorption by fat tissue. However, a causal relation might also be possible, because lutein has been linked to adipocyte differentiation, and through this mechanism lutein might reduce abdominal adiposity ${ }^{(8)}$.
Bioavailability of lutein depends on many other dietary factors, such as the presence of dietary fibre (which inhibits lutein uptake) $)^{(9)}$ and the presence of dietary fat (which increases lutein uptake $)^{(10)}$. Dietary factors might, however, not only cause interactions but also might confound the relationship between lutein intake and cardiometabolic health. In addition, because there is a high amount of lutein in vegetables, high lutein intake might reflect the overall healthy diet, or even healthy lifestyle in general, and it is, thus, important to take other dietary and lifestyle factors into account.

We recently reviewed the literature about the relationship between lutein and cardiometabolic health and observed that, despite these suggested positive effects of lutein in adults, studies in children are scarce (ETM Leermakers, SKL Darweesh, CP Baena, EM Moreira, D Melo van Lent, MJ Tielemans, T Muka, A Vitezova, R Chowdhury, WM Bramer, JC Kiefte-de Jong, JF Felix and $\mathrm{OH}$ Franco, unpublished results). To our knowledge,

Abbreviations: DBP, diastolic blood pressure; SBP, systolic blood pressure; SDS, SD score.

* Corresponding author: J. C. K. de Jong, email j.c.kiefte-dejong@erasmusmc.nl 
only one article has been published so far that related lutein to cardiometabolic health in children $^{(11)}$. In this cross-sectional study among 1339 US adolescents, lutein levels were not significantly associated with metabolic syndrome diagnosis, but higher level of lutein was significantly inversely associated with a continuous score of a number of metabolic syndrome components.

It is important to study the effects of nutrition early in life as dietary behaviours track throughout the life course ${ }^{(12)}$, and therefore early interventions can have benefits to improve health later in life. In addition, there is no recommended intake for lutein at present, and studying the effects of lutein is important from a public health perspective to determine whether recommendations are required. We aimed to assess the relationship between lutein intake at 13 months of age and cardiometabolic outcomes at the age of 6 years in a prospective population-based cohort study. The second objective was to assess whether there is an interaction of lutein intake with dietary fat and fibre intake.

\section{Methods \\ Study population}

The present study is a part of The Generation R Study, a population-based prospective cohort study in Rotterdam, the Netherlands, which has previously been described in detail elsewhere $^{(13)}$. This study was approved by the Medical Ethics Committee at Erasmus MC, University Medical Center, Rotterdam (MEC 198.782/2001/31). Written informed consent was obtained from all participating mothers. We restricted our analyses to Dutch children, because the FFQ was designed and validated for dietary assessment of a Dutch population. The FFQ was implemented in a later stage of the study, and was therefore available to $71 \%$ of the total population. Children without information on dietary intake at 13 months of age ( $n$ 1775) were excluded. As blood samples were not collected from all children, the population for analysis ranged from 1305 to 2044 children, depending on the outcome of interest (Fig. 1).

\section{Dietary assessment}

At the age of 13 months (median 12.9, $95 \%$ range $12 \cdot 2,19 \cdot 2$ ), the primary caregiver of the child (mother $86.2 \%$, father $3.8 \%$, both $9.8 \%$ or other $0.2 \%$ ) completed a 211 item semi-quantitative $\mathrm{FFQ}^{(14)}$. The FFQ asked for habitual diet of the last month, thereby covering diet from the age of 12 months onwards. At the dietary assessment, only $6.8 \%$ of the children received any breast-feeding and all the children received complementary feeding. The FFQ was developed in co-operation with the division of Human Nutrition of Wageningen University, The Netherlands. The final FFQ included questions on the frequency of consumption of the food items over the last month, the serving sizes, the type of the food item and the preparation methods - for example, for the types of vegetables, it was assessed whether they were cooked or stir-fried or whether they were raw. For each type of fruit and vegetables, it was asked whether it was consumed 'Never', 'Sometimes', 'Often' or 'Always', and consumption was weighted accordingly. Based on frequencies and servings, the food items were converted into g per $d$ using

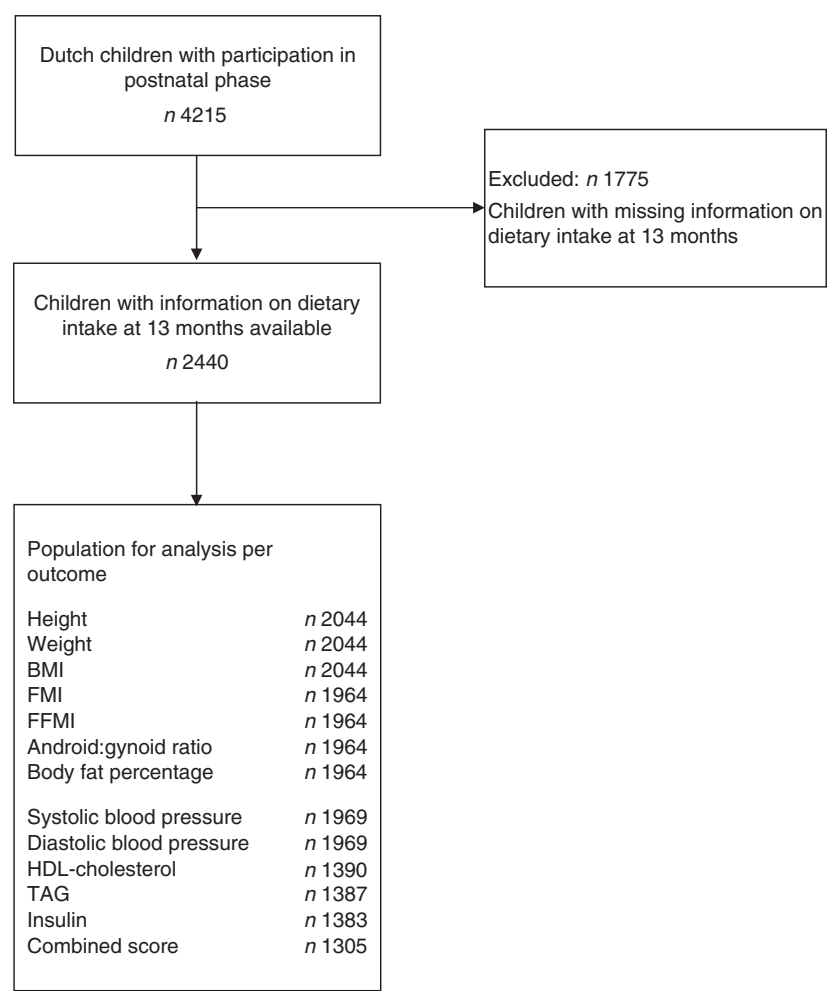

Fig. 1. Flow chart of the participants included for analysis. FMI, fat mass index; FFMI, fat-free mass index.

standardised portion sizes ${ }^{(15)}$. For all individual items of fruits and vegetables, as well as for eggs and dairy products, lutein values per gram were extracted from The Dutch Food Composition Table (NEVO) ${ }^{(16)}$. This FFQ was validated against three 24-h recalls in a representative sample of Dutch children ( $n$ 32), which showed the following intra-class correlation coefficients for macronutrients: total energy, 0.4 ; total protein, 0.7 ; total fat, 0.4; carbohydrates, 0.4; and dietary fibre, 0.7 ; and for micronutrients: calcium, 0.53 ; iron, 0.48 ; vitamin $\mathrm{C}$, 0.63; vitamin $\mathrm{D}$, 0.74 ; and vitamin $\mathrm{E}, 0.42^{(14,17)}$. In a later phase of the study, dietary lutein intake was estimated from this FFQ data, based on the Dutch Food Composition Table (NEVO) ${ }^{(16)}$, which included lutein intake from fruits and vegetables, as well as eggs and dairy products. As our objective was to assess the effects of lutein intake, we assessed lutein independent of $\beta$-carotene. The correlation between intake of lutein and intake of $\beta$-carotene was 0.94 ; thus, adjusting for $\beta$-carotene in the regression model was not possible due to multicollinearity. Therefore, we standardised lutein intake for $\beta$-carotene using the residual method $^{(18)}$, together with total energy to account for measurement error ${ }^{(19)}$. SD scores (SDS) for $(\beta$-carotene-standardised and energy-standardised) lutein intake were created and SDS of lutein intake were categorised into quartiles. The median absolute intake of lutein within each quartile was 264, 766, 1470 and $2274 \mathrm{mcg} / \mathrm{d}$, respectively.

The Supplementary material shows the results for lutein unstandardised for $\beta$-carotene (online Supplementary Tables $\mathrm{S} 1 \mathrm{~B}$ and $\mathrm{S} 2 \mathrm{~B}$ ) and the results for $\beta$-carotene individually (online Supplementary Tables S1C and S2C). 


\section{Cardiometabolic outcomes}

At a median age of 5.9 (95\% range $5.7,6.5)$ years, children visited our dedicated research facility at the Erasmus Medical Center, Sophia's Children Hospital. Weight and height were measured (without shoes and heavy clothing) using an electronic scale (SECA) and stadiometer (Holtain Limited). Total and regional fat mass was measured by Dual-energy X-ray absorptiometry scans (iDXA; General Electric, 2008) ${ }^{(20)}$. Percentage body fat was calculated as $100 \% \times$ (total body fat mass (g))/ (total body mass (fat mass + lean mass + bone mass) (g)), android:gynoid fat mass ratio was calculated as (abdominal fat mass (g)/fat mass around hips, thighs and buttocks (g)). The fatfree mass index was calculated as (lean mass $(\mathrm{kg})+$ bone mass $(\mathrm{kg})) /\left(\right.$ height $\left.^{2}(\mathrm{~m})\right)$. The fat mass index was calculated as (fat mass $(\mathrm{kg})) /\left(\right.$ height $\left.^{2}(\mathrm{~m})\right)$. Age- and sex-specific SDS were calculated for all outcomes based on the total population.

Non-fasting blood samples were drawn by antecubital venepuncture. Insulin, C-peptide, cholesterol (total, HDL and LDL) and TAG concentrations were measured using enzymatic methods (using a Cobas 8000 analyser; Roche). Quality-control samples demonstrated intra-assay and inter-assay coefficients of variation ranging from 0.69 to $1.57 \%$.

Systolic and diastolic blood pressures (SBP and DBP) were measured at the right brachial artery with the child in the supine position, using the validated automatic sphygmomanometer Datascope Accutor Plus TM ${ }^{(21)}$. Blood pressure was measured four times with 1-min intervals, and the first measurement was excluded for the calculation of mean SBP and DBP. Mean arterial pressure $(\mathrm{MAP})$ was calculated as $\mathrm{MAP}=($ mean $\mathrm{SBP}+$ $2 \times$ mean DBP)/3. In addition to the individual cardiometabolic outcomes, we calculated a continuous score following examples of previously defined metabolic syndrome scores for children $^{(22)}$, including body fat percentage, blood pressure (DBP and SBP) and serum levels of HDL-C, TAG and insulin. The cardiometabolic risk factor score was calculated as the sum of age- and sex-specific SDS of these five components, as proposed previously for paediatric populations ${ }^{(22)}$. The SDS for HDL-C were multiplied by -1 as a higher HDL-C represents a better cardiometabolic profile. The SDS for SBP and DBP were multiplied by 0.5 so that each contributed half to the blood pressure component. In summary, the cardiometabolic risk factor score was calculated as: SDS BF $\%+0.5 \times$ SDS SBP +0.5 $\times \mathrm{DBP}+$ SDS TAG $+(-1 \times$ SDS HDL-C $)+$ SDS insulin, and was standardised to facilitate interpretation of effect estimates.

\section{Covariates}

Information on maternal and paternal age, household income, educational level, maternal parity, smoking, alcohol use and folic acid use during pregnancy was obtained from questionnaires during pregnancy. Maternal and paternal weight and height were measured at enrolment and BMI was calculated $\left(\mathrm{kg} / \mathrm{m}^{2}\right)$. Information on sex, gestational age at birth and birth weight of the children was obtained from midwife and hospital registries. Information about breast-feeding, introduction of complementary feeding, history of allergy to cows' milk and any hospitalisation in 1 st year of life was derived from questionnaires at 6 and 12 months of age. The number of hours spent watching television was used as a proxy for sedentary lifestyle of the child and was derived from the questionnaire at 2 years of age. Participation in sports was used as a proxy for physical activity of the child and was derived from the questionnaire at 6 years of age. Total energy intake, food intake and nutrient intake were derived from the FFQ at 13 months. We used a modified version of the diet quality score as previously constructed by Voortman et al. ${ }^{(17)}$, including components for cereals, potatoes, fish, fats, sugar-containing beverages and snacks and candy.

\section{Statistical analysis}

Age- and sex-specific SDS were created for all outcomes. Non-normally distributed variables were transformed before standardising. Lutein was not normally disturbed, and was therefore square root transformed. We used linear regression to estimate the SD change in cardiometabolic outcomes for each quartile of lutein intake, using the lowest intake as reference category. We tested for a linear relationship using lutein intake (SDS) as a continuous variable.

Crude models were adjusted for the child's age at the dietary assessment. Multivariable models were additionally adjusted for potential confounders that were selected on the basis of the literature. The following confounders were included: maternal and paternal age and BMI, household income, maternal education, parity, smoking, alcohol and folic acid use during pregnancy, child's sex, birth weight (SDS), gestational age at birth, breast-feeding, timing of introduction to solid foods, hospitalisation in the 1st year of life, allergy to cows' milk, age at dietary assessment, total energy intake, diet quality score, watching television at age of 2 years and playing sports at the age of 6 years.

Analyses with android:gynoid fat ratio, body fat percentage or blood pressure as outcome were adjusted for child height (SDS) at the age of 6 years. We tested for interactions between lutein intake, dietary fat intake and dietary fibre intake as these factors may interact with lutein in relation to cardiometabolic health. In addition, as there may be sex differences in factors associated with childhood obesity ${ }^{(23)}$, we assessed possible heterogeneity effect by sex. These interactions were tested in the multivariable model with lutein intake (SDS) as the continuous variable.

To reduce potential bias associated with attrition, a multiple imputation procedure was performed to impute missing values on covariates ${ }^{(24)}$. Missing values on exposures and outcomes were not imputed. We used the Fully Conditional Specification method (predictive mean matching), assuming no monotone missing pattern ${ }^{(24)}$. Auxiliary variables were used to improve the model (online Supplementary material). We created ten multiply imputed data sets instead of five (default) to improve the variance. Analyses were performed in each of the ten imputed data sets separately, and the final results were pooled. Regression coefficients were pooled by taking the mean coefficient of the ten imputed data sets. The pooled standard errors were calculated according to Rubin's rules ${ }^{(25)}$. Characteristics of the children before and after multiple imputation are shown in 
the online Supplementary material. A $P$-value $<0.05$ was considered as statistically significant. Statistical analyses were performed by E. T. M. L. using SPSS, version 21.0 (SPSS Inc.).

\section{Results \\ Subject characteristics}

Median energy intake of the children at 13 months of age was $5301.1 \mathrm{~kJ} / \mathrm{d}(1267 \mathrm{kcal} / \mathrm{d})$, with a median lutein intake of $1317 \mathrm{mcg} / \mathrm{d}$ (95\% range 87, $6069 \mathrm{mcg} / \mathrm{d}$ ) (Table 1). The main contributors to lutein intake were spinach, broccoli, Brussels sprouts, green beans, curly kale, legumes and fruits, which explained in total $67 \%$ of the variation in lutein intake. All these products, except for curly kale, also significantly contributed to $\beta$-carotene intake, in addition to carrots and dairy products, and explained in total $79 \%$ of the variation in $\beta$-carotene intake.

\section{Body composition}

There were no significant associations between lutein intake and height, weight, fat mass index, fat-free mass index, android: gynoid ratio and body fat percentage. There was no significant linear association between lutein intake and BMI, and there were no consistent associations across quartiles of lutein intake (Table 2). The highest quartile of lutein intake was associated with higher BMI, and the crude associations (online Supplementary material) suggest that this increase in BMI was driven by an increase in the fat-free mass index.

\section{Cardiometabolic risk factors}

There was no association between lutein intake at 13 months of age and the cardiometabolic risk factor score at the age of 6 years (Table 3 ). In addition, there were no significant linear associations between lutein intake and any of the individual components of the cardiometabolic risk factor score, nor were there consistent associations across quartiles of lutein intake. In addition to the components of the metabolic syndrome score, we examined total cholesterol, LDL-cholesterol, C-peptide, MAP and pulse wave velocity, and found no significant associations (data not shown). Crude associations are shown in the online Supplementary material.

\section{Interactions}

We found a significant interaction between lutein intake and fat intake on HDL-cholesterol levels, but stratification for below and above the median of total fat intake showed no significant associations.

We found a significant interaction between lutein intake and fibre intake on weight. After stratification for above and below the median of fibre intake, we observed that there was a significant positive association between lutein intake and weight in the group with fibre intake below the median (increase in weight SDS 0.06 (95\% CI 0.02, 0.11)).

We also found significant sex interactions for weight, for body fat percentage and diastolic blood pressure. Stratification for sex showed that there was a significant positive association between lutein intake and weight in girls (0.06 SD (95\% CI 0.02, $0 \cdot 11)$ ), but not in boys. Stratification for sex showed no significant associations between lutein intake and body fat percentage or diastolic blood pressure in either boys or girls. There were no significant interactions between any of the other outcomes.

\section{Discussion}

In a population-based prospective cohort study, we assessed whether lutein intake at 13 months of age was associated with cardiometabolic outcomes at the age of 6 years and whether there could be an interaction of lutein intake with sex, dietary fat intake and dietary fibre intake. We observed that there were no consistent associations between lutein intake in toddlers and a cardiometabolic risk factor score at the age of 6 years, nor with any of the individual cardiometabolic risk factors. In addition, there were no consistent interactions with factors that may influence cardiometabolic risk or bioavailability of lutein such as sex, dietary fat intake and dietary fibre intake.

Our findings that lutein was not associated with cardiometabolic health is different from those from previous studies, although all the studies were conducted in older populations. Previous studies in adults have shown that higher lutein intake was associated with lower risk of metabolic syndrome ${ }^{(2-6)}$. Only one of these studies assessed dietary intake ${ }^{(4)}$, and all the other studies used blood levels of lutein. In addition, the only study in adolescents that assessed lutein intake in relation to cardiometabolic health used blood levels of lutein, and observed that higher lutein levels were associated with a lower metabolic syndrome score ${ }^{(11)}$.

As compared with the use of dietary intake levels, the use of blood levels of lutein might provide a more direct measurement of how much lutein is available. Absorption of lutein in the body depends on multiple factors, including other dietary factors such as fibre ${ }^{(9)}$ and fat ${ }^{(10)}$. In our study, we observed significant interactions of lutein intake with fibre and fat intakes with some outcomes, which could indeed be due to the fact that the bioavailability of lutein depends on dietary fibre and fat, and these factors are thus important to be taken into account in studies assessing dietary lutein. However, studies that assess blood levels of lutein already have a direct measure of the available lutein. Furthermore, when lutein is measured in the blood, other factors that affect bioavailability are taken into account as well. This is of particular benefit because there are large inter-individual differences in the bioavailability of lutein ${ }^{(26)}$, which are only partly explained by known factors that we can adjust for (e.g. age and sex), but unmeasured factors (e.g. genetic variance) might play an equally important role ${ }^{(26,27)}$. All these factors contribute to the fact that the correlation between lutein intake and lutein levels is low ${ }^{(26)}$. Dietary lutein is, therefore, not a direct marker of lutein status, which might explain why we did not observe beneficial effects of dietary intake of lutein.

However, the use of blood levels can also have challenges. As blood levels of antioxidants are being used to counteract 


\begin{tabular}{|c|c|c|c|c|c|c|c|c|}
\hline \multirow{4}{*}{$\begin{array}{l}\text { Child characteristics ( } n, \%) \\
\text { Sex (girls) }\end{array}$} & \multicolumn{8}{|c|}{ Quartiles of lutein intake } \\
\hline & \multicolumn{2}{|c|}{$\begin{array}{c}\text { Low intake } \\
\text { (median } 266 \mathrm{mcg} / \mathrm{d}) \\
n 508(24.9 \%)\end{array}$} & \multicolumn{2}{|c|}{$\begin{array}{c}\text { Mid-low intake } \\
\text { (median } 826 \mathrm{mcg} / \mathrm{d}) \\
n 512(25.0 \%)\end{array}$} & \multicolumn{2}{|c|}{$\begin{array}{c}\text { Mid-high intake } \\
\text { (median } 1486 \mathrm{mcg} / \mathrm{d}) \\
n 513(25.1 \%)\end{array}$} & \multicolumn{2}{|c|}{$\begin{array}{c}\text { High intake } \\
\text { (median } 2274 \mathrm{mcg} / \mathrm{d} \text { ) } \\
n 511(25.0 \%)\end{array}$} \\
\hline & & & & & & & & \\
\hline & 261 & 51.4 & 255 & $49 \cdot 8$ & 255 & $49 \cdot 7$ & 265 & 51.9 \\
\hline Gestational age at birth (weeks, median and $95 \%$ range) & $40 \cdot 1$ & $35 \cdot 4,42 \cdot 3$ & $40 \cdot 3$ & $35 \cdot 0,41 \cdot 3$ & $40 \cdot 1$ & $36 \cdot 5,42 \cdot 4$ & $40 \cdot 2$ & $36 \cdot 0,42 \cdot 3$ \\
\hline Birth weight ( $\mathrm{g}$, mean and sD) & 3472 & 557 & 3498 & 582 & 3519 & 523 & 3502 & 599 \\
\hline \multicolumn{9}{|l|}{ Time of introduction of solid foods (months; $n, \%$ ) } \\
\hline $0-3$ & 18 & 3.6 & 15 & 2.9 & 21 & $4 \cdot 1$ & 32 & $6 \cdot 3$ \\
\hline $3-6$ & 273 & $54 \cdot 2$ & 271 & $53 \cdot 0$ & 302 & $59 \cdot 0$ & 290 & $56 \cdot 9$ \\
\hline$>6$ & 213 & $42 \cdot \overline{3}$ & 225 & $44 \cdot 0$ & 189 & $36 \cdot 9$ & 188 & $36 \cdot 9$ \\
\hline \multicolumn{9}{|l|}{ Breast-fed $(n, \%)$} \\
\hline Never & 32 & $6 \cdot 7$ & 44 & $9 \cdot 4$ & 49 & $10 \cdot 9$ & 56 & $12 \cdot 5$ \\
\hline Partially in the first 4 months & 290 & 60.5 & 275 & $58 \cdot 8$ & 272 & 60.4 & 275 & $61 \cdot 2$ \\
\hline Exclusively for at least 4 months & 157 & $32 \cdot 8$ & 149 & 31.8 & 129 & 28.7 & 118 & $26 \cdot 3$ \\
\hline Age at dietary assessment (months, median and $95 \%$ range) & $12 \cdot 8$ & $12 \cdot 2,19 \cdot 0$ & $12 \cdot 8$ & $12 \cdot 2,18 \cdot 7$ & $12 \cdot 9$ & $12 \cdot 3,19 \cdot 0$ & $13 \cdot 0$ & $12 \cdot 3,19 \cdot 7$ \\
\hline Total energy intake $(\mathrm{kJ} / \mathrm{d}$, median and $95 \%$ range) & 5271.8 & 2916.2, 8799 & $5209 \cdot 1$ & $3037 \cdot 6,8815 \cdot 7$ & $5414 \cdot 1$ & $3020 \cdot 8,8476 \cdot 8$ & $5322 \cdot 0$ & $3389 \cdot 0,8727 \cdot 8$ \\
\hline Total energy intake (kcal/d, median and $95 \%$ range) & 1260 & 697,2103 & 1245 & 726,2107 & 1294 & 722, 2026 & 1272 & 810,2086 \\
\hline Total dietary fibre intake ( $\mathrm{g} / \mathrm{d}$, median and $95 \%$ range) & $14 \cdot 2$ & $7 \cdot 5,28 \cdot 2$ & 14.5 & $7 \cdot 0,24 \cdot 0$ & $14 \cdot 7$ & $7 \cdot 3,23 \cdot 8$ & $15 \cdot 2$ & $9 \cdot 2,24 \cdot 6$ \\
\hline Total fat intake ( $\mathrm{g} / \mathrm{d}$, median and $95 \%$ range $)$ & 37.3 & $16 \cdot 8,83 \cdot 2$ & 37.8 & $17 \cdot 8,74.5$ & 39.4 & $18 \cdot 7,80 \cdot 0$ & 39.0 & $19.5,78.5$ \\
\hline Total $\beta$-carotene intake ( $\mathrm{mcg} / \mathrm{d}$, median and $95 \%$ range) & 513 & 100,7333 & 1085 & 226,5009 & 1476 & 444,4529 & 1756 & 690,3854 \\
\hline Age at visit to research centre (months, median and $95 \%$ range) & 71 & 68,78 & 71 & 68,77 & 71 & 68,79 & 71 & 68,80 \\
\hline Weight ( $\mathrm{kg}$, median and $95 \%$ range) & $21 \cdot 6$ & $17 \cdot 3,29 \cdot 0$ & $22 \cdot 1$ & $17 \cdot 2,28 \cdot 5$ & $22 \cdot 0$ & $17 \cdot 8,28 \cdot 6$ & $21 \cdot 8$ & $17 \cdot 8,29 \cdot 9$ \\
\hline Height (cm, median and $95 \%$ range) & 118 & 108,129 & 118 & 110,129 & 119 & 109,129 & 118 & 109,129 \\
\hline $\mathrm{BMI}\left(\mathrm{kg} / \mathrm{m}^{2}\right.$, median and $95 \%$ range $)$ & $15 \cdot 6$ & $13.7,18.5$ & $15 \cdot 7$ & $13 \cdot 6,19 \cdot 1$ & $15 \cdot 6$ & $13 \cdot 6,19 \cdot 0$ & $15 \cdot 7$ & $13 \cdot 7,19 \cdot 2$ \\
\hline $\mathrm{FMl}\left(\mathrm{kg} / \mathrm{m}^{2}\right.$, median and $95 \%$ range $)$ & 3.5 & $2 \cdot 4,6 \cdot 0$ & 3.5 & $2 \cdot 4,6.4$ & 3.6 & $2 \cdot 3,6 \cdot 4$ & 3.5 & $2 \cdot 3,6 \cdot 3$ \\
\hline FFMI $\left(\mathrm{kg} / \mathrm{m}^{2}\right.$, median and $95 \%$ range $)$ & 11.8 & $10.4,13.7$ & 11.9 & $10 \cdot 6,13 \cdot 6$ & 11.9 & $10 \cdot 4,13.6$ & 11.9 & $10.4,13.6$ \\
\hline Android:gynoid fat ratio (median and $95 \%$ range) & 0.23 & $0.15,0.35$ & 0.24 & $0.15,0.38$ & 0.23 & $0.15,0.36$ & 0.23 & $0.16,0.37$ \\
\hline Total body fat percentage $(\%$, median and $95 \%$ range $)$ & 23.0 & $16.8,33.9$ & $23 \cdot 1$ & $16.4,34.5$ & $23 \cdot 3$ & $15 \cdot 9,33.9$ & $23 \cdot 2$ & $16 \cdot 1,34.5$ \\
\hline $\mathrm{HDL}$-cholesterol (mmol/l, median and $95 \%$ range) & 1.3 & $0.8,2 \cdot 1$ & 1.3 & $0.8,2 \cdot 1$ & 1.3 & $0.8,2 \cdot 0$ & 1.3 & $0.8,2 \cdot 1$ \\
\hline TAG (mmol//, median and $95 \%$ range) & 1.0 & $0.4,2.5$ & 1.0 & $0.4,2 \cdot 4$ & 0.9 & $0 \cdot 4,2 \cdot 1$ & 1.0 & $0.4,2.4$ \\
\hline Insulin (root transformed) (pmol//, median and $95 \%$ range) & $10 \cdot 8$ & $4 \cdot 3,19 \cdot 4$ & $10 \cdot 9$ & $4.5,19 \cdot 8$ & $10 \cdot 0$ & $4 \cdot 6,19 \cdot 1$ & 10.8 & $3 \cdot 7,20 \cdot 6$ \\
\hline Systolic blood pressure ( $\mathrm{mmHg}$, median and $95 \%$ range) & 101 & 88,119 & 100 & 87,121 & 102 & 88,121 & 101 & 89,117 \\
\hline Diastolic blood pressure (mmHg, median and $95 \%$ range) & 60 & 47,73 & 60 & 47,72 & 60 & 48,74 & 59 & 49,73 \\
\hline \multicolumn{9}{|l|}{ Maternal characteristics (mean and SD) } \\
\hline Maternal age (years) & 32.5 & 4.3 & $32 \cdot 3$ & 3.9 & 31.6 & 4.0 & 31.4 & 4.2 \\
\hline Maternal BMI at intake $\left(\mathrm{kg} / \mathrm{m}^{2}\right)$ & 3.8 & $24 \cdot 2$ & 3.6 & 23.9 & 4.3 & $24 \cdot 3$ & 4.2 & 24.2 \\
\hline \multicolumn{9}{|l|}{ Educational level $(n, \%)$} \\
\hline Lower & 151 & $30 \cdot 2$ & 152 & 29.9 & 196 & 38.5 & 184 & $36 \cdot 4$ \\
\hline Higher & 349 & 68.7 & 356 & $70 \cdot 1$ & 313 & 61.5 & 321 & $63 \cdot 6$ \\
\hline \multicolumn{9}{|l|}{ Net household income (Euros/month; $n, \%$ ) } \\
\hline$<2200$ & 77 & $17 \cdot 3$ & 80 & $17 \cdot 7$ & 98 & $22 \cdot 6$ & 108 & $23 \cdot 7$ \\
\hline$>2200$ & 369 & $82 \cdot 7$ & 372 & $82 \cdot 3$ & 336 & 77.4 & 347 & $76 \cdot 3$ \\
\hline \multicolumn{9}{|l|}{ Folic acid use $(n, \%)$} \\
\hline Never & 20 & $5 \cdot 3$ & 27 & $7 \cdot 0$ & 33 & 8.6 & 31 & 7.9 \\
\hline Periconceptionally & 247 & $65 \cdot 3$ & 238 & $61 \cdot 7$ & 243 & $63 \cdot 1$ & 247 & $63 \cdot 3$ \\
\hline Started in first 10 weeks & 111 & 29.4 & 121 & $31 \cdot 3$ & 109 & $28 \cdot 3$ & 112 & 28.7 \\
\hline \multicolumn{9}{|l|}{ Smoking in pregnancy $(n, \%)$} \\
\hline Never during pregnancy & 364 & $81 \cdot 1$ & 389 & $82 \cdot 8$ & 356 & 77.4 & 363 & $76 \cdot 1$ \\
\hline Until pregnancy was known & 45 & $10 \cdot 0$ & 42 & 8.9 & 51 & $11 \cdot 1$ & 53 & $11 \cdot 1$ \\
\hline Continued in pregnancy & 40 & 8.9 & 39 & $8 \cdot 3$ & 53 & 11.5 & 61 & $12 \cdot 8$ \\
\hline
\end{tabular}

FMI, fat mass index; FFMI, fat-free mass index. 


\section{Nes British Journal of Nutrition}

Table 2. Association of lutein intake at 13 months with body composition at the age of 6 years $\dagger$ (Regression coefficients and 95\% confidence intervals)

\begin{tabular}{|c|c|c|c|c|c|c|c|c|c|c|c|c|c|c|}
\hline & \multicolumn{2}{|c|}{$\begin{array}{c}\text { Height } \\
n 2044 \\
\text { SD change } \\
(95 \% \mathrm{Cl})\end{array}$} & \multicolumn{2}{|c|}{$\begin{array}{c}\text { Weight } \\
n 2044 \\
\text { SD change } \\
(95 \% \mathrm{Cl})\end{array}$} & \multicolumn{2}{|c|}{$\begin{array}{c}\text { BMI } \\
n 2044 \\
\text { SD change } \\
(95 \% \mathrm{Cl})\end{array}$} & \multicolumn{2}{|c|}{$\begin{array}{c}\text { FMl } \\
n 1964 \\
\text { SD change } \\
(95 \% \mathrm{Cl})\end{array}$} & \multicolumn{2}{|c|}{$\begin{array}{c}\text { FFMI } \\
n 1964 \\
\text { SD change } \\
(95 \% \mathrm{Cl})\end{array}$} & \multicolumn{2}{|c|}{$\begin{array}{c}\text { AG ratio } \\
n 1964 \\
\text { SD change } \\
(95 \% \mathrm{Cl})\end{array}$} & \multicolumn{2}{|c|}{$\begin{array}{c}\text { Body fat }(\%) \\
n 1964 \\
\text { SD change } \\
(95 \% \mathrm{Cl})\end{array}$} \\
\hline & $b$ & $95 \% \mathrm{Cl}$ & $b$ & $95 \% \mathrm{Cl}$ & $b$ & $95 \% \mathrm{Cl}$ & $b$ & $95 \% \mathrm{Cl}$ & $b$ & $95 \% \mathrm{Cl}$ & $b$ & $95 \% \mathrm{Cl}$ & $b$ & $95 \% \mathrm{Cl}$ \\
\hline Q1 low intake & & Ref. & & Ref. & & Ref. & & Ref. & & Ref. & & Ref. & & Ref. \\
\hline Q2 & 0.08 & $-0.03,0.19$ & 0.08 & $-0.00,0.17$ & 0.08 & $-0.01,0.17$ & 0.04 & $-0.04,0.13$ & 0.06 & $-0.04,0.17$ & 0.04 & $-0.07,0.14$ & 0.03 & $-0.07,0.12$ \\
\hline Q3 & 0.10 & $-0.02,0.20$ & 0.08 & $-0.01,0.16$ & 0.05 & $-0.03,0.14$ & 0.02 & $-0.06,0.11$ & 0.07 & $-0.04,0.17$ & -0.05 & $-0.15,0.06$ & 0.00 & $-0.09,0.09$ \\
\hline Q4 high intake & 0.02 & $-0.09,0.13$ & 0.07 & $-0.02,0.16$ & $0.09^{*}$ & $0.00,0.18$ & 0.02 & $-0.06,0.11$ & 0.09 & $-0.02,0.20$ & -0.02 & $-0.12,0.09$ & 0.00 & $-0.09,0.10$ \\
\hline Continuous (per SD) & 0.01 & $-0.03,0.05$ & 0.02 & $-0.01,0.06$ & 0.03 & $-0.00,0.06$ & 0.01 & $-0.02,0.04$ & 0.02 & $-0.02,0.06$ & -0.01 & $-0.04,0.03$ & 0.01 & $-0.03,0.04$ \\
\hline
\end{tabular}

AG, android:gynoid; FMI, fat mass index; FFMI, fat-free mass index; Ref., reference; SDS, sD score.

${ }^{*} P<0.05$.

† Linear regression coefficients reflect the difference in outcome (age- and sex-specific SDS) for mid-low, mid-high and high intake, as compared with low intake of energy and $\beta$-carotene-adjusted lutein intake.

Continuous model reflects the difference in outcome (age- and sex-specific SDS) per SD increase of energy and $\beta$-carotene-adjusted lutein intake. Models are adjusted for maternal and paternal age and BMI, household income, maternal education, parity, smoking, alcohol and folic acid use during pregnancy, child sex, birth weight (SDS), gestational age at birth, breast-feeding, timing of introduction of solids, hospitalisation in the 1st year of life, allergy to cows' milk, age at dietary assessment, total energy intake, diet quality score, television watching at age 2 years and playing sports at age 6 years. Models for android:gynoid ratio and body fat percentage are additionally adjusted for child height.

Table 3. Association of lutein intake at 13 months of age with cardiometabolic outcomes at the age of 6 years (Regression coefficients and $95 \%$ confidence intervals)

\begin{tabular}{|c|c|c|c|c|c|c|c|c|c|c|c|c|}
\hline & \multicolumn{2}{|c|}{$\begin{array}{c}\text { Systolic blood pressure } \\
n 1969 \\
\text { SD change } \\
(95 \% \mathrm{Cl})\end{array}$} & \multicolumn{2}{|c|}{$\begin{array}{c}\text { Diastolic blood pressure } \\
n 1969 \\
\text { SD change } \\
(95 \% \mathrm{Cl})\end{array}$} & \multicolumn{2}{|c|}{$\begin{array}{l}\text { HDL-cholesterol } \\
n 1390 \\
\text { SD change } \\
(95 \% \mathrm{Cl})\end{array}$} & \multicolumn{2}{|c|}{$\begin{array}{c}\text { TAG } \\
n 1387 \\
\text { SD change } \\
(95 \% \mathrm{Cl})\end{array}$} & \multicolumn{2}{|c|}{$\begin{array}{c}\text { Insulin } \\
n 1383 \\
\text { SD change } \\
(95 \% \mathrm{Cl})\end{array}$} & \multicolumn{2}{|c|}{$\begin{array}{c}\text { Cardiometabolic risk factor score } \\
n 1305 \\
\text { SD change } \\
(95 \% \mathrm{Cl})\end{array}$} \\
\hline & $b$ & $95 \% \mathrm{Cl}$ & $b$ & $95 \% \mathrm{Cl}$ & $b$ & $95 \% \mathrm{Cl}$ & $b$ & $95 \% \mathrm{Cl}$ & $b$ & $95 \% \mathrm{Cl}$ & $b$ & $95 \% \mathrm{Cl}$ \\
\hline Q1 low intake & & ef. & & ef. & & Ref. & & Ref. & & Ref. & & \\
\hline Q2 & -0.02 & $-0.14,0.10$ & -0.06 & $-0.17,0.06$ & -0.07 & $-0.22,0.08$ & -0.06 & $-0.20,0.09$ & -0.05 & $-0.20,0.10$ & 0.01 & $-0.13,0.15$ \\
\hline Q3 & 0.10 & $-0.02,0.22$ & 0.03 & $-0.08,0.15$ & 0.04 & $-0.11,0.19$ & $-0 \cdot 22^{* *}$ & $-0.37,-0.07$ & -0.08 & $-0.23,0.07$ & -0.10 & $-0.24,0.05$ \\
\hline Q4 high intake & -0.01 & $-0.14,0.11$ & -0.04 & $-0.16,0.08$ & 0.02 & $-0.13,0.17$ & 0.00 & $-0.15,0.16$ & -0.02 & $-0.17,0.13$ & -0.07 & $-0.21,0.07$ \\
\hline Continuous (per SD) & -0.01 & $-0.05,0.03$ & -0.01 & $-0.05,0.03$ & 0.02 & $-0.04,0.07$ & -0.03 & $-0.09,0.02$ & -0.01 & $-0.07,0.04$ & -0.04 & $-0.09,0.01$ \\
\hline
\end{tabular}

Ref., reference; SDS, sD score.

${ }^{* *} P<0.01$

$\dagger$ Linear regression coefficients reflect the difference in outcome (age- and sex-specific SDS) for mid-low, mid-high and high intake, as compared with low intake of energy and $\beta$-carotene-adjusted lutein intake.

Continuous model reflects the difference in outcome (age- and sex-specific SDS) per SD increase of energy and $\beta$-carotene-adjusted lutein intake. Models are adjusted for maternal and paternal age and BMI, household income, maternal education, parity, smoking, alcohol and folic acid use during pregnancy, child sex, birth weight (SDS), gestational age at birth, breast-feeding, timing of introduction of solids, hospitalisation in the 1st year of life, allergy to cows' milk, age at dietary assessment, total energy intake, diet quality score, television watching at age 2 years and playing sports at age 6 years. Models for systolic and diastolic blood pressure are additionally adjusted for child height. 
oxidative stress, disease processes with high oxidative stress may result in depletion of lutein levels, and lower levels of lutein in metabolic disorders might thus be a result of these processes ${ }^{(28)}$. This reverse causation is of particular concern in cross-sectional studies, when there could already be an active disease process. However, in pre-clinical stages of diseases, there might already be increased oxidative stress, and thus lower levels of antioxidants. For example, it has been shown that subjects with obesity or metabolic syndrome have higher levels of oxidised LDL, a marker of oxidative stress, that might deplete antioxidant levels ${ }^{(29)}$. Moreover, it is important to address this topic in studies with sufficiently long follow-up periods. Studies assessing lutein in relation to metabolic syndrome were all cross-sectional, and thus the inverse relationships that were observed could potentially be explained by reverse causation. Prospective studies in adults that investigated lutein (levels or intake) in relation to risk of type 2 diabetes found inconsistent results ${ }^{(30-33)}$, and these studies all had a follow-up period of $>10$ years, and are thus less prone to risk of reverse causation.

The large sample size and the prospective longitudinal design are important strengths of our study. In addition, we were able to adjust for a wide range of potential confounders such as socio-economic and other dietary and lifestyle factors. It is important to note that all studies on lutein in relation metabolic syndrome and diabetes in adults were observational. Besides issues with reverse causation, observational studies are subject to confounding. As nutritional and lifestyle factors are clustered $^{(34)}$, and intakes of nutrients are highly correlated, it is difficult to conclude from observational studies that any beneficial effect that is found is attributable to one specific nutrient. In the case of lutein, it could also be that not only lutein was responsible for the observed effect but also another carotenoid, the group of carotenoids as a whole, or other nutrients, foods or nutritional factors that are correlated with lutein intake could have been responsible. Indeed, we found in our study that $\beta$-carotene and lutein were highly correlated. In addition to dietary factors, socio-economic and lifestyle variables such as sedentary behaviour and physical activity are highly related to diet, and could often not be fully adjusted for in these studies.

Another possible explanation for our null findings is that we were unable to detect a relevant difference in our study. It may be argued that associations between lutein and health are easier to detect in studies where extremely low lutein intake is present among participants, and that our study had negative findings due to a relatively high lutein intake in our population. Unfortunately, there are no dietary recommendations regarding lutein intake to establish whether children have sufficient intake. Moreover, there are no other studies on lutein intake in young children in relation to cardiometabolic health, but lutein intake in our study was comparable with a study on 6-year-old Canadian children, which studied lutein in relation to cognitive function $^{(35)}$. In addition, it can be argued that diet in early life may be subject to large changes. However, we used ranking instead of absolute values, and it has been shown that diet during early life tracks into later life ${ }^{(36-38)}$

We assessed lutein intake with the use of an FFQ, and therefore measurement error in dietary intake may be present ${ }^{(19)}$. Although the FFQ that we used showed good validation against 24-h recalls for some micronutrients, we have no validation measure for lutein specifically, and we did not have blood levels of lutein to assess the correlation with estimated intake of lutein. We standardised lutein intake for total energy using the residual method ${ }^{(18)}$, which reduces the magnitude of systematic measurement error ${ }^{(19)}$. However, random error may still be present. For example, we had information on preparation method (cooking, stir-frying) for vegetables, but no information on cooking time. Moreover, lutein values might vary within products. For example, nutritional values of eggs are influenced by the diet of the hens ${ }^{(39)}$. As this random error generally leads to bias towards the null, this could, therefore, have led to an underestimation of the true effect ${ }^{(40)}$.

We found significant interactions of lutein with fat intake, fibre intake and sex on several outcomes, but not on all. We might have been underpowered to detect possible interactions on other outcomes; therefore, the results should be interpreted with caution. In addition, we looked at the interaction with total fat or fibre intake, although a possible interaction might depend on which point in time they are consumed.

Finally, for this analysis, we included only Dutch children with complete nutritional data and a visit to the research centre at the age of 6 years. Although this approach reduces heterogeneity of the study population and related measurement of diet, it may decrease the generalisability of our results.

In conclusion, although earlier studies in adults did show a beneficial effect of lutein on cardiometabolic health, we found no association between lutein intake at the age of 1 year and cardiometabolic outcomes in children at 6 years of age. However, this was the first study in this age-group, and further studies are needed to elucidate the role of lutein in young children.

\section{Acknowledgements}

The Generation R Study is conducted by the Erasmus Medical Center in close collaboration with the School of Law and Faculty of Social Sciences of the Erasmus University Rotterdam, the Municipal Health Service Rotterdam area, the Rotterdam Homecare Foundation and the Stichting Trombosedienst and Artsenlaboratorium Rijnmond (STAR). The authors gratefully acknowledge the contribution of participating mothers and children, general practitioners, hospitals, midwives and pharmacies in Rotterdam.

The Generation R Study was made possible by the financial support from the Erasmus Medical Center, Rotterdam, the Erasmus University Rotterdam and the Netherlands Organization for Health Research and Development. V. W. V. J. received an additional grant from the Netherlands Organization for Health Research and Development (ZonMw - VIDI 016.136.361). E. T. M. L., O. H. F. and J. C. K.-d J. work in ErasmusAGE, a centre for ageing research across the life course funded by Nestlé Nutrition (Nestec Ltd), Metagenics Inc. and AXA. Nestlé Nutrition (Nestec Ltd), Metagenics Inc. and AXA had no role in design and conduct of the study, collection, management, analysis and interpretation of the data as well as preparation, review or approval of the manuscript. 
E. T. M. L., J. C. K.-d. J., A. H., V. W. V. J. and O. H. F. designed the research. E. T. M. L., J. C. K.-d. J., A. H., V. W. V. J. and O. H. F. conducted the research. A. H., V. W. V. J. and O. H. F. provided essential materials. E. T. M. L. analysed the data. E. T. M. L. and J. C. K.-d. J. wrote the paper. J. C. K.-d. J. had primary responsibility for the final content. All the authors read and approved the final version of the manuscript.

There are no conflicts of interest.

\section{Supplementary material}

For supplementary material/s referred to in this article, please visit http://dx.doi.org/doi:10.1017/S0007114515002779

\section{References}

1. Calvo MM (2005) Lutein: a valuable ingredient of fruit and vegetables. Crit Rev Food Sci 45, 671-696.

2. Beydoun MA, Shroff MR, Chen X, et al. (2011) Serum antioxidant status is associated with metabolic syndrome among U.S. adults in recent national surveys. J Nutr 141, 903-913.

3. Coyne T, Ibiebele TI, Baade PD, et al. (2009) Metabolic syndrome and serum carotenoids: findings of a cross-sectional study in Queensland, Australia. Br J Nutr 102, 1668-1677.

4. Sluijs I, Beulens JWJ, Grobbee DE, et al. (2009) Dietary carotenoid intake is associated with lower prevalence of metabolic syndrome in middle-aged and elderly men. J Nutr $\mathbf{1 3 9}$, 987-992.

5. Sugiura M, Nakamura M, Ogawa K, et al. (2008) Associations of serum carotenoid concentrations with the metabolic syndrome: interaction with smoking. Br J Nutr 100, 1297-1306.

6. Suzuki K, Ito Y, Inoue T, et al. (2011) Inverse association of serum carotenoids with prevalence of metabolic syndrome among Japanese. Clin Nutr 30, 369-375.

7. Waters D, Clark RM, Greene CM, et al. (2007) Change in plasma lutein after egg consumption is positively associated with plasma cholesterol and lipoprotein size but negatively correlated with body size in postmenopausal women. $J$ Nutr 137, 959-963.

8. Gunanti IR, Marks GC, Al-Mamun A, et al. (2014) Low serum concentrations of carotenoids and vitamin e are associated with high adiposity in Mexican-American children. J Nutr 144, 489-495.

9. Yeum KJ \& Russell RM (2002) Carotenoid bioavailability and bioconversion. Ann Rev Nutr 22, 483-504

10. Roodenburg AJ, Leenen R, van het Hof KH, et al. (2000) Amount of fat in the diet affects bioavailability of lutein esters but not of alpha-carotene, beta-carotene, and vitamin $\mathrm{E}$ in humans. Am J Clin Nutr 71, 1187-1193.

11. Beydoun MA, Canas JA, Beydoun HA, et al. (2012) Serum antioxidant concentrations and metabolic syndrome are associated among U.S. adolescents in recent national surveys. J Nutr 142, 1693-1704.

12. Mikkila V, Rasanen L, Raitakari OT, et al. (2005) Consistent dietary patterns identified from childhood to adulthood: the cardiovascular risk in young finns study. Br J Nutr 93, 923-931.

13. Jaddoe VWV, van Duijn CM, Franco OH, et al. (2012) The Generation R Study: design and cohort update 2012. Eur J Epidemiol 27, 739-756.

14. Kiefte-de Jong JC, de Vries JH, Bleeker SE, et al. (2013) Sociodemographic and lifestyle determinants of 'western-like' and 'health conscious' dietary patterns in toddlers. Br J Nutr $\mathbf{1 0 9}$ $137-147$.
15. Donders-Engelen M, Heijden van der L \& Hulshof KF (2003) Maten, gewichten en codenummers (Measures, Weights and Code Numbers). Wageningen: Human Nutrition of TNO and Wageningen University.

16. RIVM (2011) NEVO-tabel; Nederlands Voedingsstoffenbestand 2011 (Dutch Food Composition Database). The Hague: RIVM/Voedingscentru.

17. Voortman T, Kiefte-de Jong JC, Geelen A, et al. (2015) The development of a diet quality score for preschool children and its validation and determinants in the Generation $\mathrm{R}$ Study. J Nutr 145, 306-314.

18. Willett WC, Howe GR \& Kushi LH (1997) Adjustment for total energy intake in epidemiologic studies. Am J Clin Nutr 65 , $1220-1228$

19. Kipnis V, Subar AF, Midthune D, et al. (2003) Structure of dietary measurement error: results of the OPEN biomarker study. Am J Epidemiol 158, 14-21.

20. Kaul S, Rothney MP, Peters DM, et al. (2012) Dual-energy $\mathrm{X}$-ray absorptiometry for quantification of visceral fat. Obesity (Silver Spring) 20, 1313-1318.

21. Wong SN, Sung RYT \& Leung LCK (2006) Validation of three oscillometric blood pressure devices against auscultatory mercury sphygmomanometer in children. Blood Press Monit 11, 281-291.

22. Eisenmann JC (2008) On the use of a continuous metabolic syndrome score in pediatric research. Cardiovasc Diabetol 7, 17.

23. Govindan M, Gurm R, Mohan S, et al. (2013) Gender differences in physiologic markers and health behaviors associated with childhood obesity. Pediatrics 132, 468-474.

24. Sterne JAC, White IR, Carlin JB, et al. (2009) Multiple imputation for missing data in epidemiological and clinical research: potential and pitfalls. BMJ 338, b2393.

25. Rubin DB \& Schenker N (1991) Multiple imputation in healthcare databases - an overview and some applications. Stat Med 10, 585-598.

26. Gruber M, Chappell R, Millen A, et al. (2004) Correlates of serum lutein plus zeaxanthin: findings from the Third National Health and Nutrition Examination Survey. J Nutr 134, 2387-2394.

27. Borel P, Desmarchelier C, Nowicki M, et al. (2014) Interindividual variability of lutein bioavailability in healthy men: characterization, genetic variants involved, and relation with fasting plasma lutein concentration. Am J Clin Nutr 100, 168-175.

28. Ford ES, Mokdad AH, Giles WH, et al. (2003) The metabolic syndrome and antioxidant concentrations - findings from the Third National Health and Nutrition Examination Survey. Diabetes 52, 2346-2352.

29. Van Guilder GP, Hoetzer GL, Greiner JJ, et al. (2006) Influence of metabolic syndrome on biomarkers of oxidative stress and inflammation in obese adults. Obesity (Silver Spring) 14, 2127-2131

30. Hozawa A, Jacobs DR, Steffes MW, et al. (2006) Associations of serum carotenoid concentrations with the development of diabetes and with insulin concentration: interaction with smoking - The Coronary Artery Risk Development in Young Adults (CARDIA) Study. Am J Epidemiol 163, 929-937.

31. Kataja-Tuomola MK, Kontto JP, Mannisto S, et al. (2011) Intake of antioxidants and risk of type 2 diabetes in a cohort of male smokers. Eur J Clin Nutr 65, 590-597.

32. Montonen J, Knekt P, Jarvinen R \& Reunanen A (2004) Dietary antioxidant intake and risk of type 2 diabetes. Diabetes Care 27, 362-366.

33. Wang L, Liu SM, Pradhan AD, et al. (2006) Plasma lycopene, other carotenoids, and the risk of type 2 diabetes in women. Am J Epidemiol 164, 576-585.

34. Leech RM, McNaughton SA \& Timperio A (2014) The clustering of diet, physical activity and sedentary behavior in children and adolescents: a review. Int J Behav Nutr Phy 11, 4. 
35. Mulder KA, Innis SM, Rasmussen BF, et al. (2014) Plasma lutein concentrations are related to dietary intake, but unrelated to dietary saturated fat or cognition in young children. J Nutr Sci $\mathbf{3}$, e11.

36. Craigie AM, Lake AA, Kelly SA, et al. (2011) Tracking of obesity-related behaviours from childhood to adulthood: a systematic review. Maturitas 70, 266-284.

37. Northstone K \& Emmett PM (2008) Are dietary patterns stable throughout early and mid-childhood? A birth cohort study. BrJ Nutr 100, 1069-1076.
38. Schwartz C, Scholtens PAMJ, Lalanne A, et al. (2011) Development of healthy eating habits early in life. Review of recent evidence and selected guidelines. Appetite 57, 796-807.

39. Leeson S \& Caston L (2004) Enrichment of eggs with lutein. Poultry Sci 83, 1709-1712.

40. Kipnis V \& Freedman LS (2008) Impact of exposure measurement error in nutritional epidemiology. J Natl Cancer I 100, 1658-1659. 